\title{
Efficacy of recombinant human soluble thrombomodulin for acute exacerbation of idiopathic pulmonary fibrosis: A systematic review and meta-analysis
}

\author{
BAOJUN WANG ${ }^{1}$ and TING $\mathrm{LI}^{2}$ \\ ${ }^{1}$ Department of Critical Care Medicine, Xinchang County Hospital of Traditional Chinese Medicine, Shaoxing, \\ Zhejiang 312500; ${ }^{2}$ Department of Science and Education, Huzhou Hospital of Traditional Chinese Medicine \\ Affiliated to Zhejiang University of Traditional Chinese Medicine, Huzhou, Zhejiang 313000, P.R. China
}

Received November 6, 2019; Accepted March 11, 2020

DOI: $10.3892 /$ etm.2020.8709

\begin{abstract}
Idiopathic pulmonary fibrosis (IPF) is a chronic fibrosing lung disease of unknown etiology. Recombinant human soluble thrombomodulin (rhTM) is used for the management of acute exacerbation (AE) of IPF. The present review aimed to summarize the evidence and perform a meta-analysis of the efficacy and safety of rhTM in the management of AE-IPF. An electronic search of titles and abstracts published until 31st August 2019 was performed in the PubMed, Biomed Central, Scopus and Embase databases. Studies comparing rhTM-treated and control subjects with AE-IPF and assessing mortality and adverse events were included. Six studies met the inclusion criteria. A total of 145 patients received rhTM, while 146 patients served as controls. The meta-analysis indicated that rhTM resulted in a reduction in 28-day [odds ratio (OR), 0.25; 95\% CI, 0.08-0.77; $\left.\mathrm{P}=0.02 ; \mathrm{I}^{2}=0 \%\right]$ and 90-day mortality $(\mathrm{OR}, 0.29 ; 95 \% \mathrm{CI}$, $\left.0.17-0.49 ; \mathrm{P}<0.00001 ; \mathrm{I}^{2}=0 \%\right)$ compared with the controls Adverse events were pooled and no difference was determined between rhTM and control groups (OR, 1.07; 95\% CI, $\left.0.45-2.51 ; \mathrm{P}=0.88 ; \mathrm{I}^{2}=0 \%\right)$. It was indicated that administration of rhTM may reduce the short-term mortality in patients with AE-IPF; however, the quality of evidence was not high. The drug appears to be safe without any enhanced risk of adverse events, although high-quality randomized controlled trials with a large sample size are required to further support its use in the treatment of IPF.
\end{abstract}

Correspondence to: Dr Ting Li, Department of Science and Education, Huzhou Hospital of Traditional Chinese Medicine Affiliated to Zhejiang University of Traditional Chinese Medicine, 315 South Street, Huzhou, Zhejiang 313000, P.R. China

E-mail: seven7_star@163.com

Key words: survival, pulmonary fibrosis, acute exacerbation, thrombomodulin, adverse events

\section{Introduction}

Idiopathic pulmonary fibrosis (IPF) is a chronic fibrosing lung disease of unknown etiology (1). IPF is associated with an increased fibroblast population, leading to excessive deposition of collagen in the lung alveolar spaces $(1,2)$. This pathological change subsequently leads to decreased gas exchange and respiratory failure. While disease progression is usually slow, a number of patients experience acute deterioration of respiratory function, which is termed as acute exacerbation (AE) of IPF (AE-IPF) (2). In a retrospective review of 461 patients with IPF in South Korea, the 1- and 3-year incidences of AE-IPF were estimated to be 14.2 and $20.7 \%$, respectively (3). While the exact etiology of AE-IPF remains elusive, the prognosis is poor, with a systematic review estimating the mortality rate to be $60 \%$ at 1 month and $67 \%$ at 3 months after disease onset (4).

The pathophysiology of AE-IPF has been attributed to procoagulant and antifibrinolytic activity in affected patients (3). Imokawa et al (5) demonstrated the strong presence of tissue factor, a coagulation initiator, in the lung tissues of patients with IPF. Kotani et al (6) have reported higher concentrations of plasminogen activator inhibitor (PAI)-1 and PAI-2 antigen levels in bronchoalveolar lavage (BAL) supernatant fluids and cell lysates, suggestive of an antifibrinolytic activity. While one study corroborate this theory based on improved survival of patients treated with low-molecular-weight heparin (7), another study indicated a lack of benefit of anticoagulant therapy in such patients (8). High-dose corticosteroids and immunosuppressive drugs, including cyclosporine A, have also been used for managing AE-IPF, but the clinical efficacy of these drugs is still under dispute $(9,10)$.

Thrombomodulin, a transmembranous protein expressed on the surface of vascular endothelial cells, is an essential component in the regulation of intravascular coagulation (11). Due to its anticoagulant and anti-inflammatory effects, thrombomodulin has been successfully used in the management of disseminated intravascular coagulation (DIC) (11). Recombinant human soluble thrombomodulin (rhTM; Recomodulin; Asahi Kasei Pharma Corp.), which comprises only the extracellular domain of thrombomodulin, has also been explored in the management of various 
respiratory, renal and cardiovascular diseases (12). Recently, several trials reported on the use of rhTM in the management of AE-IPF (13-15). While research is still at the nascent stage, there is a requirement to summarize the results of these multiple studies to evaluate the quality of evidence and provide a direction for further research. Therefore, the purpose of the present systematic review and meta-analysis was to evaluate the efficacy and safety of rhTM when used in the management of AE-IPF.

\section{Materials and methods}

Guidelines. The guidelines of the Preferred Reporting Items for Systematic Reviews and Meta-analyses (16) and the Cochrane Handbook for Systematic Reviews of Intervention (17) were followed during the preparation of this review.

Search strategy. An electronic search of titles and abstracts in the PubMed (https://pubmed.ncbi.nlm.nih.gov), Biomed Central (https://www.biomedcentral.com), Scopus (https:// www.scopus.com/home.uri) and Embase (https://www. embase.com/login\#search) databases was performed using the following key words: 'Recombinant human thrombomodulin', 'thrombomodulin', 'pulmonary fibrosis', 'AE', 'IPF' and 'clinical outcomes'. Titles were searched by two independent reviewers (BW and TL) with the last search performed on 31st August 2019. The reference lists of published studies and review articles on the subject were also searched for the identification of any further trials. After screening records by their titles and abstracts, the full texts of selected articles were retrieved. Each of the two reviewers assessed individual studies based on the inclusion criteria. Disagreements, if any, were resolved by discussion.

Study selection and outcomes. Using the common evidence medicine framework, population, intervention, comparison, outcome (18), all types of studies performed on patients with AE-IPF (Population) were included. Trials comparing rhTM (Intervention) vs. control (Comparison) for AE-IPF and assessing mortality and adverse events (Outcomes) were included in the review. Duplicate studies based on the same dataset, non-comparative studies, case series, case reports, review articles and studies that were not published in English language were excluded. In the case of studies with overlapping datasets, the study encompassing the largest dataset was selected.

Two independent reviewers retrieved data from selected studies using a standardized data collection form. The details sourced were the following: Authors, publication year, country of origin, sample size, baseline characteristics of study participants, treatment protocol, mortality and adverse events. The primary outcome of interest was the incidence of 28- and 90- day mortality. The secondary outcome was the incidence of adverse events.

Risk of bias assessment. The Risk of Bias Assessment Tool for Non-randomized Studies was used for quality assessment (19). Studies were rated as having low, high or unclear risk of bias regarding the following points: Selection of participants, confounding variables, measurement of exposure blinding of
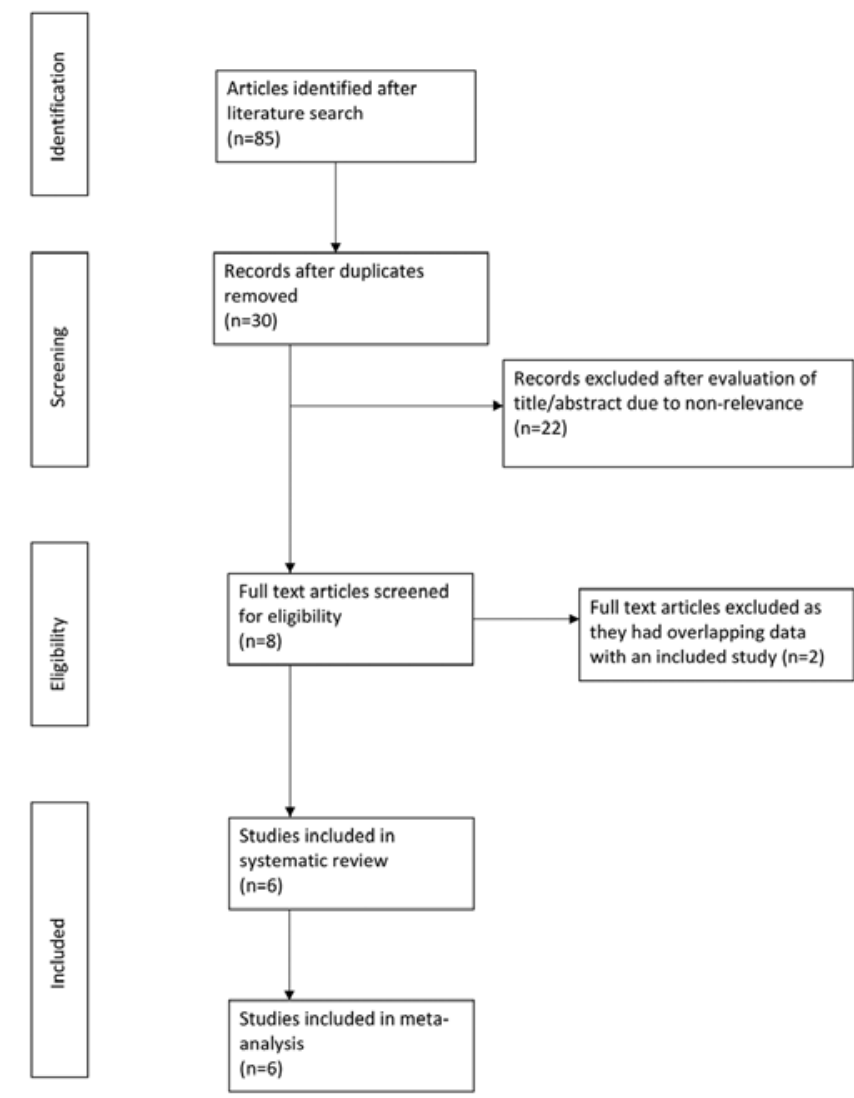

Figure 1. Study flow-chart.

outcome assessment, incomplete outcome data and selective outcome reporting.

Statistical analysis. RevMan version 5.3 (Cochrane Collaboration) was used for meta-analysis. Considering the methodological heterogeneity amongst studies, a random-effects model was used to calculate the pooled effect size. Data were summarised using the Mantel-Haenszel odds ratio (OR) with 95\% CI. Inter-study heterogeneity was estimated using the $\mathrm{I}^{2}$ statistic. $\mathrm{I}^{2}$ values of $25-50 \%$ were considered to indicate low heterogeneity, $50-75 \%$ medium heterogeneity and $>75 \%$ represented substantial heterogeneity.

\section{Results}

Literature search and study selection. The literature search yielded a total of 85 records. After the exclusion of duplicates, 30 unique articles were identified. The titles and abstracts of these 30 articles were then screened for possible inclusion in the present study. A total of 22 articles were excluded due to non-relevance and full texts of 8 articles were retrieved (Fig. 1). A total of three studies (19-21) had overlapping datasets, of which two $(20,21)$ were excluded. A total of six studies (13-15, 21-23) were finally included in the present systematic review and meta-analysis.

Features of the studies included. Details of the studies included are presented in Table I. Two studies were retrospective studies $(13,22)$ and three were single-arm prospective studies 


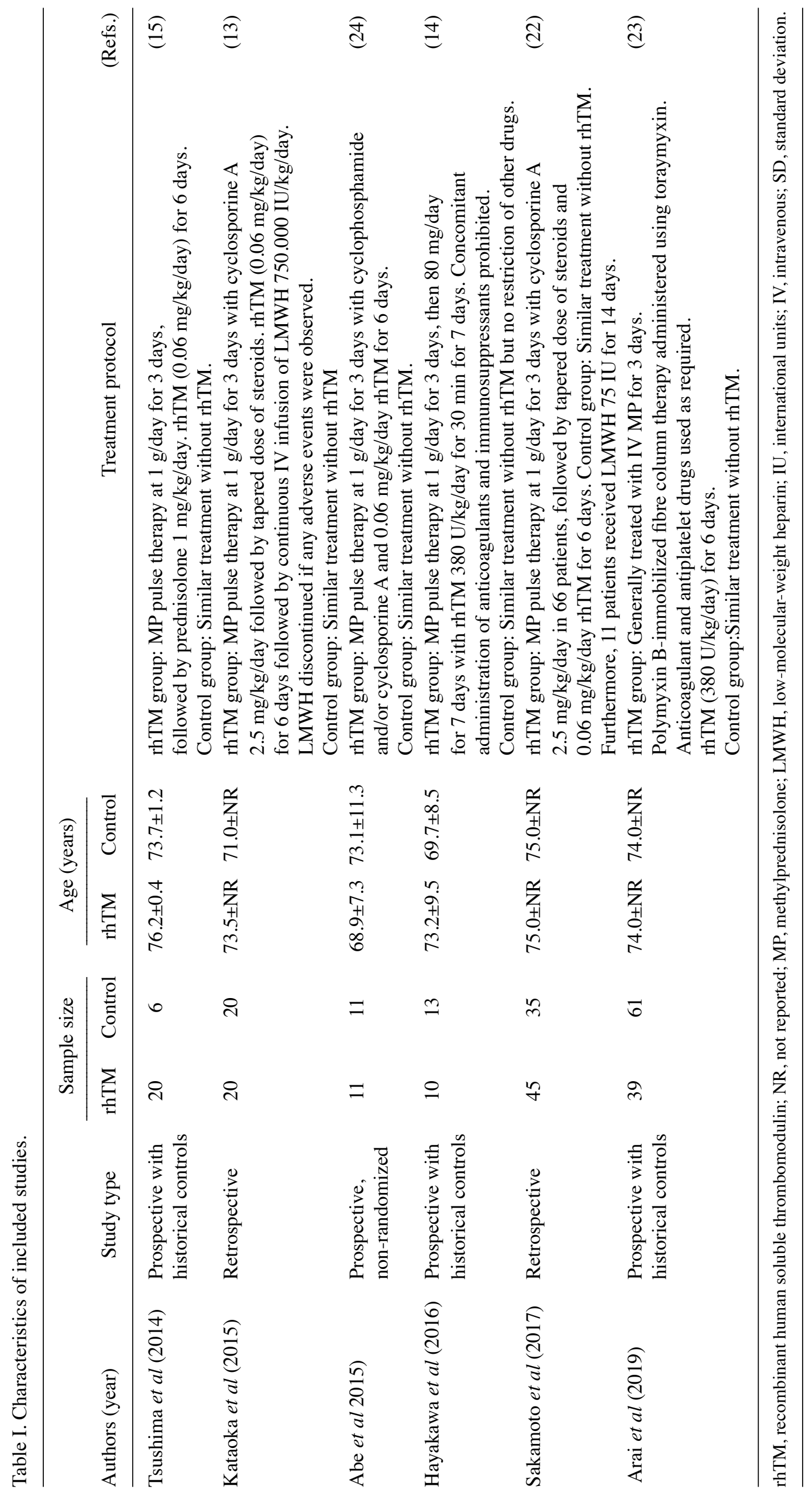




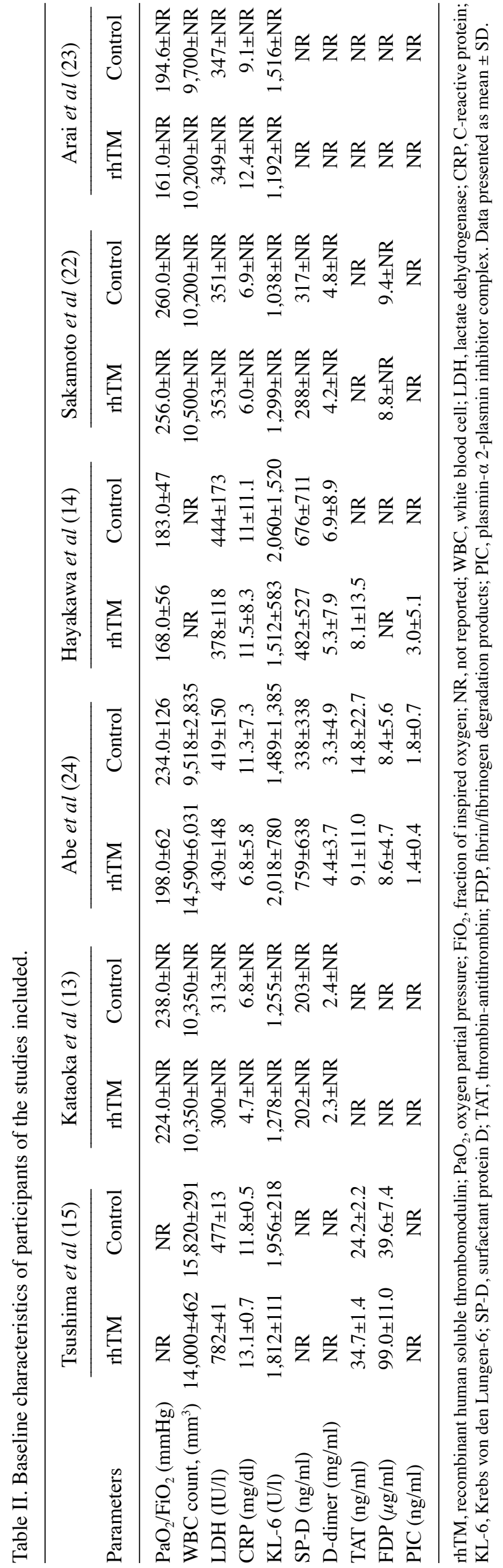

utilizing a historical control group $(14,15,23)$, while one was a non-randomized prospective trial (24). All trials were performed in Japan. The sample size varied across the studies, with 6-61 participants per group. The baseline parameters of the experimental and control groups as reported by the studies are presented in Table II. The authors reported negligible differences in the baseline parameters between the rhTM and control groups. The dosage of rhTM was the same across the studies $(0.06 \mathrm{mg} / \mathrm{kg} / \mathrm{day}$ is equivalent to $380 \mathrm{U} / \mathrm{kg} / \mathrm{day})$. Methylprednisolone pulse therapy was administered for three days in all studies in the experimental and control groups.

Outcomes. The 28-day mortality rates were reported by three studies $(14,15,24)$. Within the rhTM group, $29.26 \%$ of patients died, while $56.66 \%$ of patients died in the control group. The meta-analysis indicated that treatment with rhTM was associated with a significant reduction in 28-day mortality compared with that in the control group (OR, $0.25 ; 95 \% \mathrm{CI}$, 0.08-0.77; P=0.02; $\mathrm{I}^{2}=0 \%$; Fig. 2).

The 90-day mortality rates were reported by five $(13,14,21-23)$ of the six studies included. A total of 125 patients received rhTM, while 140 patients served as controls. The overall 90 -day mortality rate was $33.6 \%$ in the rhTM group and $62.85 \%$ in the control group. Pooled analysis indicated that rhTM significantly reduced the 90-day mortality in patients with AE-IPF (OR, 0.29; 95\% CI, 0.17-0.49; $\mathrm{P}<0.00001 ; \mathrm{I}^{2}=0 \%$; Fig. 3).

Data on adverse events were reported by five trials (13,14,21-23). Details of adverse events in the studies included are presented in Table III. Hayakawa et al (14) did not report any adverse events, while Sakamoto et al (22) reported only minor adverse events in the study group, which did not require discontinuation of treatment. Abe et al (24) reported one case of hepatic and renal failure in the study group. The authors suspected the cause of death in this patient to be a drug interaction between warfarin and cyclosporine, leading to uncontrolled serum levels of cyclosporine and subsequent renal and hepatic failure. Kataoka et al (13) reported hemorrhagic complications in the study and control groups, which were managed by discontinuation of rhTM and low-molecular-weight heparin, respectively. Arai et al (23) reported two cases of bleeding (1 case of gastrointestinal and 1 case of nasal) in the control group but no hemorrhagic complication in the rhTM group. All adverse events were pooled for a meta-analysis, which demonstrated no significant difference between the rhTM and control groups (OR, 1.07; 95\% CI, 0.45-2.51; $\mathrm{P}=0.88 ; \mathrm{I}^{2}=0 \%$; Fig. 4).

Risk of bias. The assessment of the risk of bias in the included studies is presented in Table IV. A high risk of bias was noted across all studies in the selection of participants, which were recruited at different time intervals in all trials.

\section{Discussion}

Several different therapies have been studied to improve outcomes in AE-IPF. These include corticosteroids, anticoagulants, antacids, ambrisentan, imatinib, nintedanib, phosphodiesterase-5 inhibitors, pirfenidone, dual endothelin receptor antagonists (e.g. macitentan) and combination therapy 
Table III. Adverse events reported by the studies included.

\begin{tabular}{|c|c|c|c|}
\hline Study & $\operatorname{rhTM}$ & Control & (Ref.) \\
\hline Tsushima et al & NR & NR & (15) \\
\hline Kataoka et al & $\begin{array}{l}\text { One case of hemosputum on day } 5 \text {. } \\
\text { and one case of acute DVT on day } 4 \text {. }\end{array}$ & $\begin{array}{l}\text { One case of bleeding from central venous catheter } \\
\text { on day } 3 \text { and } 1 \text { case of subcutaneous bleeding } \\
\text { on day } 8 .\end{array}$ & (13) \\
\hline Abe et al & $\begin{array}{l}\text { One patient died of hepatic and renal failure } \\
\text { due to high concentration of cyclosporine } \\
\text { (suspected drug interaction with warfarin). }\end{array}$ & None. & (24) \\
\hline Hayakawa et al & None. & None. & (14) \\
\hline Sakamoto et al & $\begin{array}{l}\text { Mild hemoptysis and hematuria in one patient } \\
\text { on day } 1 . \text { No case of serious bleeding. }\end{array}$ & None. & (22) \\
\hline Arai et al & $\begin{array}{l}\text { One case of gastrointestinal bleeding, } \\
\text { one case of nasal bleeding, one case of DVT, } \\
\text { one case of cerebral infarction, } \\
\text { one case of drug-induced hepatitis, } \\
1 \text { case of an unspecified vascular event, } \\
\text { one case of fungal infection. }\end{array}$ & $\begin{array}{l}\text { Two cases of pneumomediastinum, one case of } \\
\text { pneumothorax, three cases of DVT, one case of } \\
\text { hepatitis B, one case of hepatitis B PCR positive, } \\
\text { one case of sulfamethoxazole/trimethoprim, } \\
\text {-associated thrombocytopenia, one case of MRSA } \\
\text { pneumonia, one case of fungal infection. }\end{array}$ & (23) \\
\hline
\end{tabular}

rhTM, recombinant human soluble thrombomodulin; NR, not reported; DVT, deep vein thrombosis; MRSA, methicillin-resistant Staphylococcus aureus.

Table IV. Risk of bias summary.

\begin{tabular}{|c|c|c|c|c|c|c|c|}
\hline Study & $\begin{array}{c}\text { Selection } \\
\text { of } \\
\text { participants }\end{array}$ & $\begin{array}{c}\text { Confounding } \\
\text { variables }\end{array}$ & $\begin{array}{l}\text { Measurement } \\
\text { of exposure }\end{array}$ & $\begin{array}{l}\text { Blinding of } \\
\text { outcome } \\
\text { assessment }\end{array}$ & $\begin{array}{c}\text { Incomplete } \\
\text { outcome } \\
\text { data }\end{array}$ & $\begin{array}{l}\text { Selective } \\
\text { outcome } \\
\text { reporting }\end{array}$ & (Ref.) \\
\hline Tsushima et al & High risk & High risk & Low risk & Low risk & Low risk & Low risk & (15) \\
\hline Kataoka et al & High risk & Low risk & Low risk & Low risk & Low risk & Low risk & (13) \\
\hline Abe et al & High risk & Low risk & Low risk & Low risk & Low risk & Low risk & (24) \\
\hline Hayakawa et al & High risk & High risk & Low risk & Low risk & Low risk & Low risk & (14) \\
\hline Sakamoto et al & High risk & Low risk & Low risk & Low risk & Low risk & Low risk & (22) \\
\hline Arai et al & High risk & Low risk & Low risk & Low risk & Low risk & Low risk & (23) \\
\hline
\end{tabular}

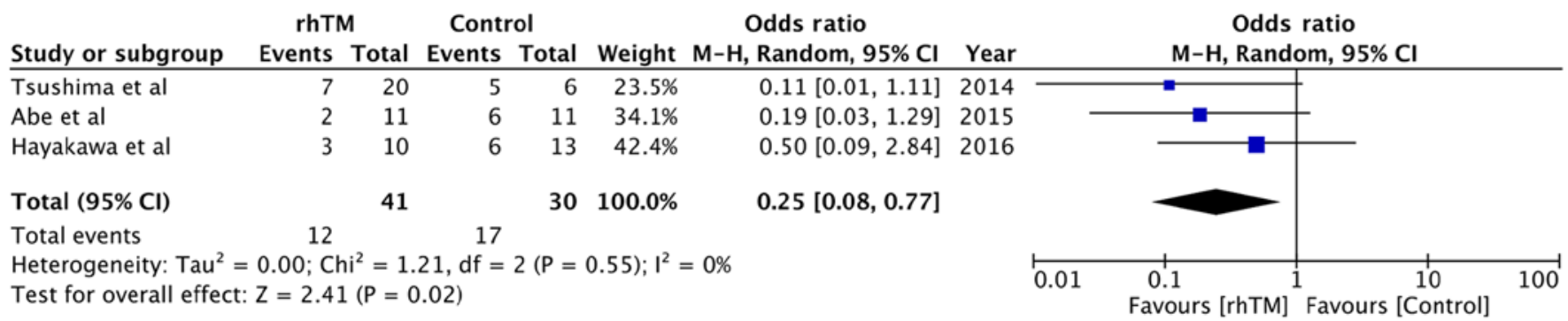

Figure 2. Forest plot for comparison of 28-day mortality between the rhTM and control groups. The black diamond denotes the point estimate and $95 \%$ CIs of the pooled data and indicates a 75\% reduced odds of mortality with rhTM treatment. rhTM, recombinant human soluble thrombomodulin; df, degrees of freedom, M-H, Mantel-Haenszel.

of prednisone, azathioprine and $\mathrm{N}$-acetylcysteine (25). Despite several attempted treatment strategies, no single management protocol has been accepted as the standard to date. The updated American Thoracic Society/European Respiratory Society/ Japanese Respiratory Society/Latin American Thoracic
Society guidelines do not recommend the use of warfarin, ambrisentan, imatinib or the combination therapy of prednisone, azathioprine and $\mathrm{N}$-acetylcysteine, while providing a conditional recommendation for nintedanib, pirfenidone, macitentan and phosphodiesterase-5 inhibitors (25). 


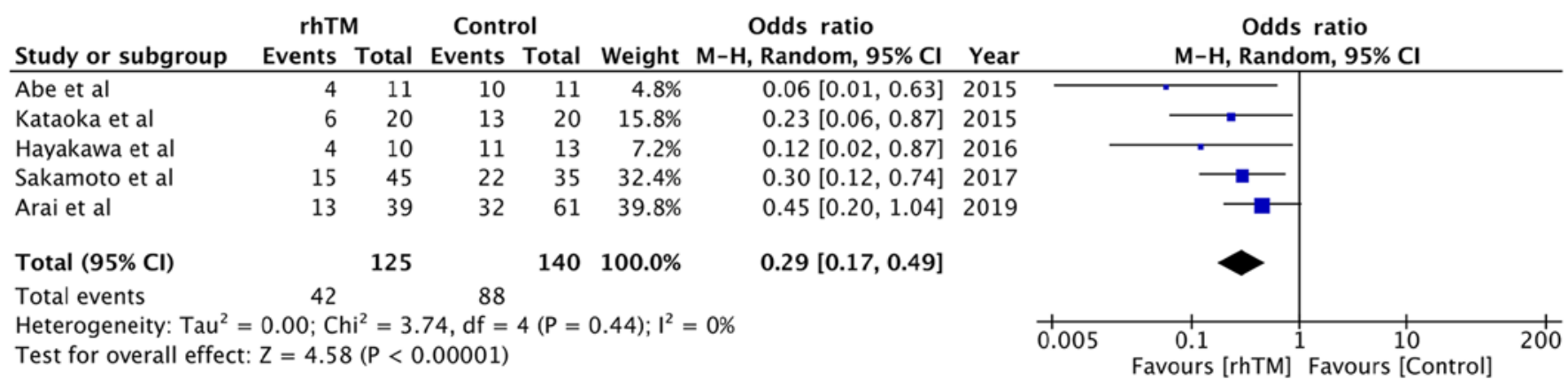

Figure 3. Forest plot for comparison of 90-day mortality between rhTM and control groups. The black diamond denotes the point estimate and 95\% CI of the pooled data and indicates a $71 \%$ reduced odds of mortality with rhTM treatment. rhTM, recombinant human soluble thrombomodulin; df, degree of freedom; M-H, Mantel-Haenszel.

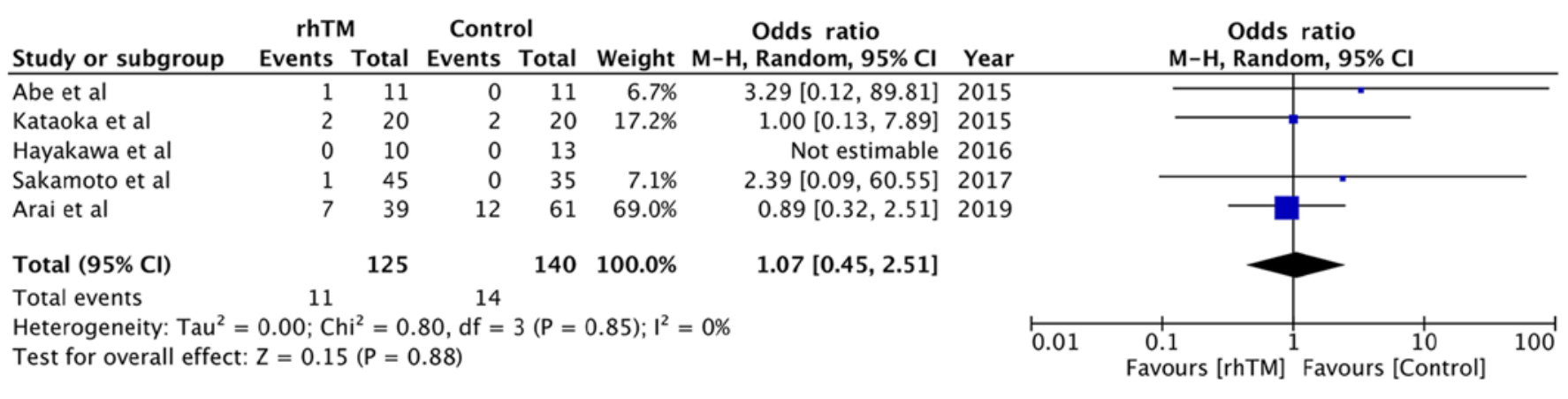

Figure 4. Forest plot for comparison of adverse events between rhTM and control groups. The black diamond denotes the point estimate and 95\% CI of the pooled data and indicates that the odds of adverse events with rhTM was 1.07-fold of that in the control group, with no statistical significance. rhTM, recombinant human soluble thrombomodulin; df, degree of freedom; M-H, Mantel-Haenszel.

The use of immunosuppressants, including cyclophosphamides, cyclosporine or tacrolimus, has been examined, but only in small retrospective studies providing inconclusive evidence for the potential benefits of these drugs $(9,26)$. In routine practice, administration of corticosteroids in the form of methylprednisolone pulse therapy is the most commonly employed management technique, despite low evidence supporting its use (27). Corticosteroid therapy was utilized in all six of the included studies of the present review but with disparity in the use of immunosuppressant drugs. Cyclophosphamide and/or cyclosporine were administered in three studies in the experimental and control groups, while in the clinical trial by Hayakawa et al (14), only the control group received immunosuppressants. The variance in the treatment strategies for AE-IPF may be attributed to the poorly understood pathophysiological mechanism of the disease.

In an attempt to elucidate the underlying pathology of AE, Collard et al (28) compared the plasma biomarker profiles between patients with AE-IPF, acute lung injury and stable IPF. The results indicated a significant increase in the levels of type II alveolar epithelial cell injury/proliferation markers [Krebs von den Lungen-6 (KL-6) and surfactant protein D], endothelial cell injury markers (von Willebrand factor), inflammation markers (interleukin-6) and coagulation markers (protein $\mathrm{C}$, thrombomodulin and PAI-1) in patients with AE-IPF. Kataoka et al (13) detected high levels of D-dimer and thrombomodulin in BAL fluid from patients with AE-IPF. Song et al (3) reported that increased levels of C-reactive protein are a poor prognostic factor for AE-IPF. These studies suggested that dysfunction of endothelial cells, coagulation abnormalities and inflammation have an important role in the pathophysiology of AE-IPF.

The dual anticoagulant and antiinflammatory action of rhTM has been utilized in the management of AE-IPF (13). rhTM activates protein $\mathrm{C}$ by the formation of reversible complexes with thrombin. These complexes inactivate coagulation factors, thereby downregulating intravascular coagulation (23). Inflammation is also suppressed by inhibition of neutrophil adhesion, improved endothelial barrier function, decreased complement activation and expression of inflammatory cytokines, inactivation of bradykinin C3a and C5a and increased high-mobility group box-1 degradation $(12,29)$.

Following a systematic literature search, six unique studies evaluating the efficacy and safety of rhTM in AE-IPF were identified. The dose of rhTM was the same across studies (0.06 mg/kg/day is equivalent to $380 \mathrm{U} / \mathrm{kg} /$ day) (30). All studies utilized a 6-day drug protocol with the exception of one study (14), in which the drug was administered for seven days. With the limited evidence, the difference between the 6- and 7-day rhTM protocol remains to be determined. After pooling the data of 145 patients in the study group and 146 patients in the control group, the present results indicated that administration of rhTM decreased the 90-day mortality by $71 \%$ (CI, 51-83\%) compared with that in patients who were not treated with the drug. Similarly, the 28-day mortality was reduced by $75 \%$ (CI, 23-92\%) with the administration of rhTM, albeit with a large CI, probably due to the small number of studies with limited sample size included in the 
present analysis. The results of the present study are similar to a recently published meta-analysis of Kamiya et al (31). In their analysis of four studies, the authors noted a statistical significant difference in 90 day mortality with the use of rhTM in AE-IPF. However, due to fewer studies, no meta-analysis was conducted for 28 day mortality in their study.

The survival rate in the control arm of the present analysis was similar to that in studies utilizing only corticosteroid therapy for the management of $\operatorname{AE}-\operatorname{IPF}(3,4)$. It is important to note that to date, no randomized controlled trial (RCT) has been performed and the present review is a pooled analysis of non-randomized studies. The absence of randomization potentially introduces bias due to the presence of confounding factors. Mortality in AE-IPF may be dependent on several confounding factors, including disease severity, oxygen partial pressure/fraction of inspired oxygen ratio, D-dimer and KL-6 levels, high-resolution computed tomography (HRCT) pattern (diffuse/non-diffuse) and use of concomitant therapies, including anticoagulants and immunosuppressants $(3,4)$. While multivariate regression analysis performed in the clinical trials by Arai et al (23) and Sakamoto et al (22) indicated that rhTM independently improved the 90-day survival in patients with AE-IPF, the absence of robust RCTs diminishes the overall level of evidence.

Data from the post-marketing surveillance of rhTM therapy in patients with DIC demonstrated an incidence of all adverse events of $7.1 \%$, with bleeding and serious bleeding complication rates of 5.5 and $6.8 \%$, respectively (32). With similar rates of adverse events in the study (8.8\%) and control groups (10\%), the present results indicated rhTM to be safe in the management of AE-IPF. None of the included studies reported any serious bleeding events during the treatment with rhTM.

The following limitations should be considered when interpreting the results of the present review. First, as mentioned above, no RCTs were available for inclusion in the present review. The majority of the included studies used a historical control group for which the diagnosis and treatment protocols would have differed. In spite of the authors reporting baseline similarity amongst the study groups, certain confounding factors may have introduced bias in the overall results. Furthermore, the inherent limitations of included studies, e.g. methodological variation, limited sample size and differences in the use of adjunctive therapies, including anticoagulants, immunosuppressants, antibiotics and high-flow oxygen, may have led to skewed results. As another limitation, BAL and HRCT were not rigorously utilized for diagnostic purposes in all studies included. In addition, the difference in general supportive care amongst studies, as well as between the experimental and control groups, may have influenced the results. In addition, it was not possible to pool data other than those of mortality and adverse events in the present meta-analysis. The lack of evaluation of other clinical outcomes in the included studies restricted the present meta-analysis to these variables only. Finally, all studies were reported from a single country. The influence of the region and ethnicity on clinical outcomes requires to be considered.

In conclusion, bearing in mind the limitations our systematic review and meta-analysis, our results indicated that rhTM may improve the short-term mortality in patients with AE-IPF. The drug appears to be safe without any enhanced risk of adverse events. There is a requirement for high-quality
RCTs with a large sample size to provide robust evidence supporting its use.

\section{Acknowledgements}

Not applicable.

\section{Funding}

No funding was received.

\section{Availability of data and materials}

The datasets used and/or analyzed during the current study are available from the corresponding author on reasonable request.

\section{Authors' contributions}

BW conceived and designed the study. BW and TL collected the data and performed the literature search. BW was involved in the writing of the manuscript. All authors read and approved the final manuscript.

\section{Ethical approval and consent to participate}

Not applicable.

\section{Patient consent for publication}

Not applicable.

\section{Competing interests}

The authors declare that they have no competing interests.

\section{References}

1. Crystal RG, Bitterman PB, Rennard SI, Hance AJ and Keogh BA: Interstitial lung diseases of unknown cause. Disorders characterized by chronic inflammation of the lower respiratory tract (first of two parts). N Engl J Med 310: 154-166, 1984.

2. Kondoh Y, Taniguchi H, Kawabata Y, Yokoi T, Suzuki K and Takagi K: Acute exacerbation in idiopathic pulmonary fibrosis. Analysis of clinical and pathologic findings in three cases. Chest 103: 1808-1812, 1993.

3. Song JW, Hong S-B, Lim C-M, Koh Y and Kim DS: Acute exacerbation of idiopathic pulmonary fibrosis: Incidence, risk factors and outcome. Eur Respir J 37: 356-363, 2011.

4. Agarwal R and Jindal SK: Acute exacerbation of idiopathic pulmonary fibrosis: A systematic review. Eur J Intern Med 19: 227-235, 2008.

5. Imokawa S, Sato A, Hayakawa H, Kotani M, Urano $T$ and Takada A: Tissue factor expression and fibrin deposition in the lungs of patients with idiopathic pulmonary fibrosis and systemic sclerosis. Am J Respir Crit Care Med 156: 631-636, 1997.

6. Kotani I, Sato A, Hayakawa H, Urano T, Takada Y and Takada A: Increased procoagulant and antifibrinolytic activities in the lungs with idiopathic pulmonary fibrosis. Thromb Res 77: 493-504, 1995.

7. Kubo H, Nakayama K, Yanai M, Suzuki T, Yamaya M, Watanabe $\mathrm{M}$ and Sasaki H: Anticoagulant therapy for idiopathic pulmonary fibrosis. Chest 128: 1475-1482, 2005.

8. Noth I, Anstrom KJ, Calvert SB, de Andrade J, Flaherty KR, Glazer C, Kaner RJ and Olman MA; Idiopathic Pulmonary Fibrosis Clinical Research Network (IPFnet): A placebo-controlled randomized trial of warfarin in idiopathic pulmonary fibrosis. Am J Respir Crit Care Med 186: 88-95, 2012. 
9. Sakamoto S, Homma S, Miyamoto A, Kurosaki A, Fujii T and Yoshimura K: Cyclosporin A in the treatment of acute exacerbation of idiopathic pulmonary fibrosis. Intern Med 49: 109-115, 2010.

10. Ryerson CJ, Cottin V, Brown KK and Collard HR: Acute exacerbation of idiopathic pulmonary fibrosis: Shifting the paradigm. Eur Respir J 46: 512-520, 2015.

11. Ogawa Y, Yamakawa K, Ogura H, Kiguchi T, Mohri T, Nakamori Y, Kuwagata Y, Shimazu T, Hamasaki T and Fujimi S Recombinant human soluble thrombomodulin improves mortality and respiratory dysfunction in patients with severe sepsis. J Trauma Acute Care Surg 72: 1150-1157, 2012.

12. Ito T, Thachil J, Asakura H, Levy JH and Iba T: Thrombomodulin in disseminated intravascular coagulation and other critical conditions-a multi-faceted anticoagulant protein with therapeutic potential. Crit Care 23: 280, 2019.

13. Kataoka K, Taniguchi H, Kondoh Y, Nishiyama O, Kimura T, Matsuda T, Yokoyama T, Sakamoto K and Ando M: Recombinant human thrombomodulin in acute exacerbation of idiopathic pulmonary fibrosis. Chest 148: 436-443, 2015.

14. Hayakawa S, Matsuzawa Y, Irie T, Rikitake H, Okada N and Suzuki Y: Efficacy of recombinant human soluble thrombomodulin for the treatment of acute exacerbation of idiopathic pulmonary fibrosis: A single arm, non-randomized prospective clinical trial. Multidiscip Respir Med 11: 38, 2016.

15. Tsushima K, Yamaguchi K, Kono Y, Yokoyama T, Kubo K, Matsumura T, Ichimura $\mathrm{Y}$, Abe $\mathbf{M}$, Terada J and Tatsumi $\mathrm{K}$ Thrombomodulin for acute exacerbations of idiopathic pulmonary fibrosis: A proof of concept study. Pulm Pharmacol Ther 29: 233-240, 2014.

16. Moher D, Liberati A, Tetzlaff J and Altman DG; PRISMA Group: Preferred reporting items for systematic reviews and meta-analyses: The PRISMA statement. PLoS Med 6: e1000097, 2009.

17. Higgins J, Thomas J, Chandler J, Cumpston M, Li T, Page M and Welch V (eds): Cochrane handbook for systemic reviews of interventions. 2nd edition. Cochrane, 2019. John Wiley \& Sons, Inc., Hoboken NJ. https://doi.org/10.1002/9781119536604.

18. Schardt C, Adams MB, Owens T, Keitz S and Fontelo P: Utilization of the PICO framework to improve searching PubMed for clinical questions. BMC Med Inform Decis Mak 7: 16, 2007.

19. Kim SY, Park JE, Lee YJ, Seo HJ, Sheen SS, Hahn S, Jang BH and Son HJ: Testing a tool for assessing the risk of bias for nonrandomized studies showed moderate reliability and promising validity. J Clin Epidemiol 66: 408-414, 2013

20. Isshiki T, Sakamoto S, Kinoshita A, Sugino K, Kurosaki A and Homma S: Recombinant human soluble thrombomodulin treatment for acute exacerbation of idiopathic pulmonary fibrosis: A retrospective study. Respiration 89: 201-207, 2015.

21. Shimizu H, Sakamoto S, Isshiki T, Furuya K, Kurosaki A and Homma S: Association of serum high-mobility group box protein 1 level with outcomes of acute exacerbation of idiopathic pulmonary fibrosis and fibrosing nonspecific interstitial pneumonia. PLoS One 13: e0196558, 2018.

22. Sakamoto S, Shimizu H, Isshiki T, Sugino K, Kurosaki A and Homma S: Recombinant human soluble thrombomodulin for acute exacerbation of idiopathic pulmonary fibrosis: A historically controlled study. Respir Investig 56: 136-143, 2018.
23. Arai T, Kida H, Ogata Y, Marumo S, Matsuoka H, Gohma I, Yamamoto S, Mori M, Sugimoto C, Tachibana K, et al; Osaka Acute Exacerbation of Interstitial Pneumonia Research Group: Recombinant thrombomodulin for acute exacerbation in idiopathic interstitial pneumonias. Respirology 24: 658-666, 2019.

24. Abe M, Tsushima K, Matsumura T, Ishiwata T, Ichimura Y, Ikari J, Terada J, Tada Y, Sakao S, Tanabe N, et al: Efficacy of thrombomodulin for acute exacerbation of idiopathic pulmonary fibrosis and nonspecific interstitial pneumonia: A nonrandomized prospective study. Drug Des Devel Ther 9: 5755-5762, 2015.

25. Raghu G, Rochwerg B, Zhang Y, Garcia CA, Azuma A, Behr J, Brozek JL, Collard HR, Cunningham W, Homma S, et al; American Thoracic Society; European Respiratory society; Japanese Respiratory Society; Latin American Thoracic Association: An official ATS/ERS/JRS/ALAT clinical practice guideline: Treatment of idiopathic pulmonary fibrosis. An update of the 2011 clinical practice guideline. Am J Respir Crit Care Med 192: 3-19, 2015

26. Horita N, Akahane M, Okada Y, Kobayashi Y, Arai T, Amano I, Takezawa T, To M and To Y: Tacrolimus and steroid treatment for acute exacerbation of idiopathic pulmonary fibrosis. Intern Med 50: 189-195, 2011.

27. Cuerpo S, Moisés J, Hernández-González F, Benegas $M$ Ramirez J, Sánchez M, Agustí À and Sellares J: Acute exacerbations of idiopathic pulmonary fibrosis: Does clinical stratification or steroid treatment matter? Chron Respir Dis 16: 1479973119869334, 2019.

28. Collard HR, Calfee CS, Wolters PJ, Song JW, Hong SB, Brady S, Ishizaka A, Jones KD, King TE Jr, Matthay MA, et al: Plasma biomarker profiles in acute exacerbation of idiopathic pulmonary fibrosis. Am J Physiol Lung Cell Mol Physiol 299: 3-7, 2010.

29. Okamoto T, Tanigami H, Suzuki K and Shimaoka M: Thrombomodulin: A bifunctional modulator of inflammation and coagulation in sepsis. Crit Care Res Pract 2012: 614545 , 2012.

30. Hayakawa M, Kushimoto S, Watanabe E, Goto K, Suzuki Y, Kotani T, Kiguchi T, Yatabe T, Tagawa J, Komatsu F, et al: Pharmacokinetics of recombinant human soluble thrombomodulin in disseminated intravascular coagulation patients with acute renal dysfunction. Thromb Haemost 117: 851-859, 2017.

31. Kamiya $\mathrm{H}$ and Panlaqui OM: The efficacy of recombinant human soluble thrombomodulin (rhsTM) treatment for acute exacerbation of idiopathic pulmonary fibrosis: A systematic review and meta-analysis. BMC Pulm Med 20: 57, 2020

32. Eguchi Y, Gando S, Ishikura H, Saitoh D, Mimuro J, Takahashi $H$ Kitajima I, Tsuji H, Matsushita T, Tsujita R, et al: Post-marketing surveillance data of thrombomodulin alfa: Sub-analysis in patients with sepsis-induced disseminated intravascular coagulation. J Intensive Care 2: 30, 2014.

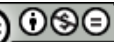

This work is licensed under a Creative Commons Attribution-NonCommercial-NoDerivatives 4.0 International (CC BY-NC-ND 4.0) License. 